\title{
Design of Wideband Elliptical Ring Monopole Antenna Using Characteristic Mode Analysis
}

\author{
Bhaskara Rao Perli1,* · Maheswara Rao Avula ${ }^{2}$
}

\begin{abstract}
This paper presents the systematic design of an elliptical ring monopole wideband antenna using characteristic mode analysis. The modal analysis is used to analyze the bandwidth and the radiation performance of the radiating patch. The resonance frequencies of three characteristic modes are close to each other with similar modal current distributions and characteristic fields. These three characteristic modes are simultaneously excited by an effective feeding technique. The proposed model achieves wideband characteristics. The proposed model is printed on an inexpensive FR4 substrate with a size of $20 \mathrm{~mm} \times 18 \mathrm{~mm} \times 1.6 \mathrm{~mm}$ and has a wide impedance bandwidth of $124.4 \%$ in the range of 3.6-15.46 GHz. The prototype has been fabricated and the measured results show good agreement with simulated results. The antenna will cover WLAN, WiMAX, Wi-Fi, and X-band applications.
\end{abstract}

Key Words: Characteristic Modes, Elliptical Ring Patch, Surface Currents, Tapered Feed, Wideband Antenna.

\section{INTRODUCTION}

Recently, microstrip patch antennas (MSAs) have become more popular and attractive in mobile communication, global positioning system, satellites, military applications, and modern wireless systems due to their exciting attributes they are lightweight, have low production costs, are low profile, have conformal configuration, and are ease to integrate and fabricate [1]. Designing antennas with wideband characteristics is an important aspect to ensuring high data rates. However, microstrip antennas intrinsically have narrow bandwidth and low gain. However, practical applications currently require wider impedance bandwidth and small antennas. Many approaches have been tried to improve the bandwidth of various microstrip antennas [2-4]. These antenna designs have been performed through simulation and experimental studies. The initial design procedure was based on engineering experience and parametric research but was not carried out systematically.

The Theory of Characteristic Modes (TCM) was initiated by Garbacz [5] in 1965 and subsequently revised by Harrington and Mautz $[6,7]$ in the 1970s. TCM can provide physical insights into the potential radiation properties of a conductive object. A conductive object naturally consists of characteristic modes $(\mathrm{CMs})$, which are calculated numerically before using the source. Each CM consists of a characteristic angle or an eigenvalue that provides information about the mode resonance and resonant behavior. CMs depend only on the shape and size of the radiation element to govern the performance of the antenna design. TCM has been involved in the design of antennas for wireless applications [8].

The potential of TCM has been successfully extended to enhance the bandwidth of antennas including the bowtie antenna

Manuscript received November 24, 2020 ; Revised March 10, 2021 ; Accepted March 29, 2021. (ID No. 20201124-184J)

${ }^{1}$ Department of Electronics and Communication Engineering, Jawaharlal Nehru Technological University, Anantapur, Andhra Pradesh, India.

${ }^{2}$ Department of Electronics and Communication Engineering, PBR Visvodaya Institute of Technology \& Science, Kavali, Andhra Pradesh, India.

*Corresponding Author: Bhaskara Rao Perli (e-mail: mail2bhaskarp@gmail.com)

This is an Open-Access article distributed under the terms of the Creative Commons Attribution Non-Commercial License (http://creativecommons.org/licenses/by-nc/4.0) which permits unrestricted non-commercial use, distribution, and reproduction in any medium, provided the original work is properly cited.

(c) Copyright The Korean Institute of Electromagnetic Engineering and Science. 
[9], an antenna with circular aperture [10], a dodecagonalshaped antenna [11], a circular ring antenna [12], and an $\mathrm{H}-$ shaped slot antenna [13]. TCM was used to design a metallic loop wideband antenna with a size of $36 \mathrm{~mm} \times 36 \mathrm{~mm} \times 0.8$ $\mathrm{mm}$, in which two CMs were combined to improve the impedance bandwidth by $51.6 \%$ [14]. A combination of a dipole and a loop antenna with a size of $80 \mathrm{~mm} \times 40 \mathrm{~mm} \times 0.8 \mathrm{~mm}$ was proposed, in which a wide impedance bandwidth reached up to 44.2\% by simultaneous excitation of the first two modes [15]. TCM was used to analyze the resonance behavior of the rectangular U-slot patch antenna with the various feed techniques, exciting the modes that contribute to the total radiation [16]. The size of the proposed model was $140.17 \mathrm{~mm} \times 134.54 \mathrm{~mm}$ $\times 7.62 \mathrm{~mm}$, which aimed to achieve a wide impedance bandwidth of $96 \%$ by exciting three CMs such as 1,3 , and 4 . These antennas have a complex feed structure to excite the desired modes and improve the antenna impedance bandwidth. The present paper aims to improve the bandwidth of a simple and small antenna structure by simultaneously exciting the first two modes with higher order modes using a simple microstrip feed technique.

\section{CHARACTERISTIC MODE ANALYSIS}

$\mathrm{CM}$ analysis is a process of solving an eigenvalue problem. The eigenvalues describe the radiation behavior of a radiating structure. Two important parameters involved in CMA for evaluating each $\mathrm{CM}$ of a given object are presented as per Chen and Wang [17].

Modal significance (MS) is represented as

$$
\mathrm{MS}=\frac{1}{\left|1+j \lambda_{n}\right|} \text {, a real quantity. }
$$

In this case, $\lambda_{n}$ is real eigenvector and $n$ is the index of the order of each mode. MS is an inherent characteristic of each mode, specifying the ability to connect each mode to external sources. It measures each mode's contribution to the total electromagnetic response regarding a particular source. In some cases, it is easier to use the MS in addition to eigenvalues to examine the resonance of a structure. Regions of the frequency spectrum with MS $>0.7$ are considered significant modes that are suitable for radiation. Ideally, the perfect radiating mode should be $\mathrm{MS}=1$ for $\lambda_{n}=0$.

Characteristic angle $(\mathrm{CA})$ is defined as

$$
C A=180^{\circ}-\tan ^{-1}\left(\lambda_{n}\right) .
$$

$\mathrm{CA}$ can provide information about the behavior of the mode near the resonance. For $\mathrm{CA}=180^{\circ}$, the mode will be resonant. $\mathrm{CA}$ values are between $90^{\circ}$ and $180^{\circ}$ or $180^{\circ}$ and $270^{\circ}$; the modes are inductive or capacitive, respectively. For a good radiator, the $\mathrm{CA}=180^{\circ}$ for $\lambda_{n}=0$.

\section{CMA OFTHE ANTENNA STRUCTURE}

The CMA of antenna structures was analyzed using a multilayer solver of the full-wave simulator. In the case of a multilayer solver, the radiating elements and ground plane were set as perfect electric conductors with a zero thickness, and the substrate component was set at loss-free with a thickness of $1.6 \mathrm{~mm}$. CMA was applied to the antenna structure without a feed port. The basic structure of antenna- 1 used for CMA is shown in Fig. 1 with the $x$-radius and the $y$-radius of the ellipse set to $5 \mathrm{~mm}$ and $6 \mathrm{~mm}$, respectively. The geometry of the antenna- 1 consisted of an ellipse-shaped patch and the partial ground plane, which were arranged on the top and bottom of the FR4 substrate.

The MS of the first six CMs is shown in Fig. 2. In this, modes 1, 2, 5, and 6 have large MS values at frequencies of 4.9, $8,14.6$, and $12 \mathrm{GHz}$; these modes are good radiators. Modes 3 and 4 have small MS values, so these modes are not good radiators. Here, surface currents are only considered for the radiating patch.

According to Fig. 3, the surface currents of the first six modes of antenna- 1 are described as follows:

- Mode 1 consists of medium current density at the bottom of the ellipse contour.

- Modes 2 and 5 have more current density on the left and

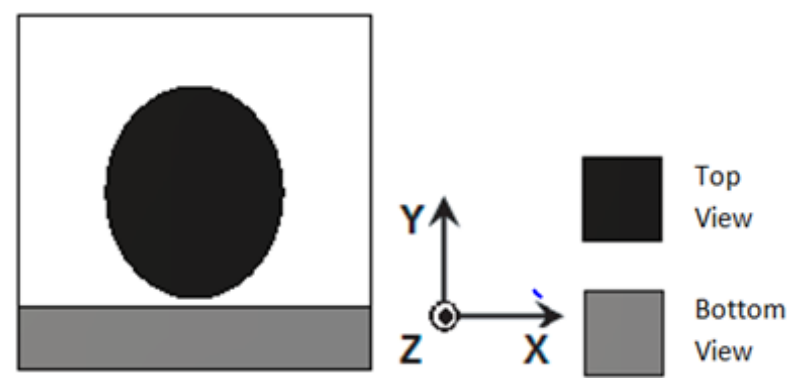

Fig. 1. Geometry of basic antenna-1.

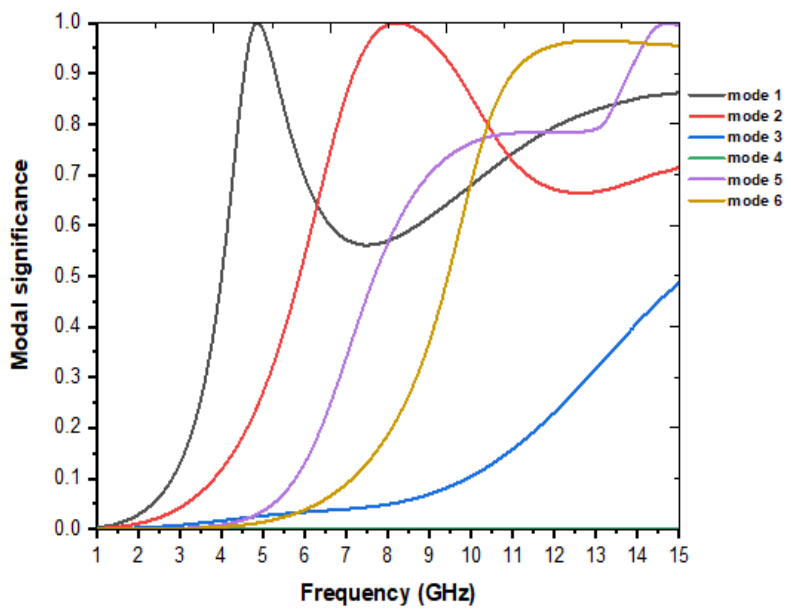

Fig. 2. Modal significance of the first six CMs of basic antenna-1. 


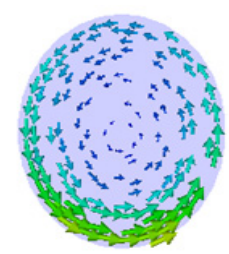

mode 1

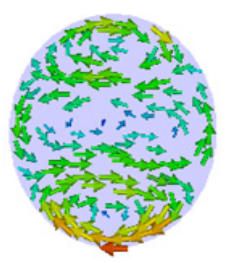

mode 4

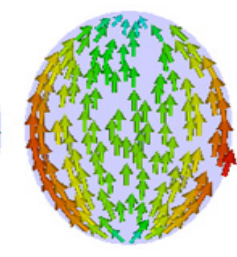

mode 2

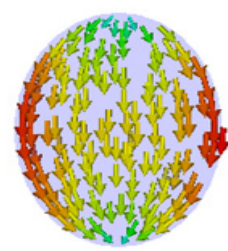

mode 5

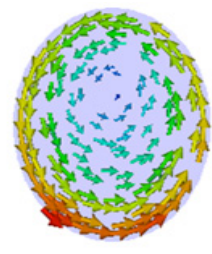

mode 3

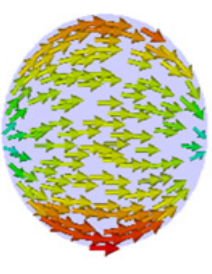

mode 6

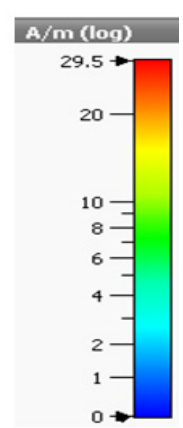

Fig. 3. Surface currents of the first six CMs of basic antenna-1.

right contours and less inside the ellipse. The surface current direction for mode 2 is vertical upward, while for mode 5 , it is the opposite. The centers of the top and bottom edges have null currents.

- Modes 3 and 4 form the current loops, indicating that these modes are not involved in radiation.

- Mode 6 has more current density on the top and bottom contour and less inside the ellipse. The surface current direction for mode 2 is horizontal. There are null currents at the centers of the left and right edges.

To reconfigure the surface currents of antenna- 1 , the dimensions of the ellipse in antenna- 1 change according to the $\mathrm{x}^{-}$and $\mathrm{y}$-radius of $8 \mathrm{~mm}$ and $4 \mathrm{~mm}$, respectively, (as shown in Fig. 4). The geometry of antenna- 2 is the same as that of antenna- 1 with a modified dimension of the ellipse-shaped patch.

Compared with Fig. 2, Fig. 5 shows that mode 1 is almost identical at $4.9 \mathrm{GHz}$; however, mode 2 shows a shift from 8 $\mathrm{GHz}$ to $9.3 \mathrm{GHz}$. In addition to modes 5 and 6 , mode 4 also has a large MS value at $14.6 \mathrm{GHz}$. Like antenna-1, mode 3 has a small MS value, so this mode is not a good radiator.

Fig. 6 describes the surface currents of the first six modes of antenna- 2 as follows:

- Mode 1 consists of medium current density at the lower edge of the ellipse contour, which corresponds to that of mode 1 in antenna-1, and nulls appear in the upper area.
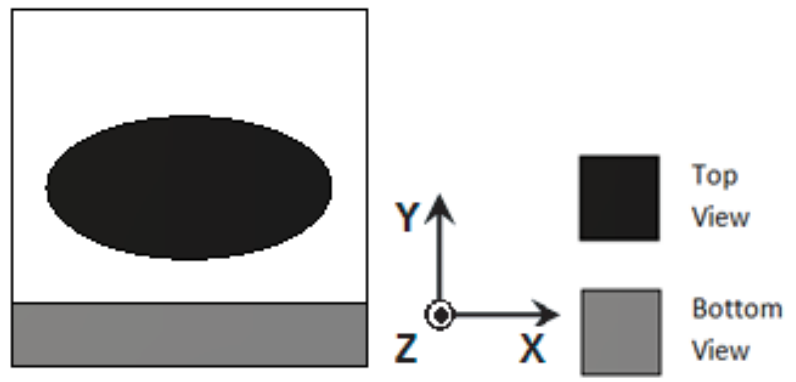

Fig. 4. Geometry of antenna-2.

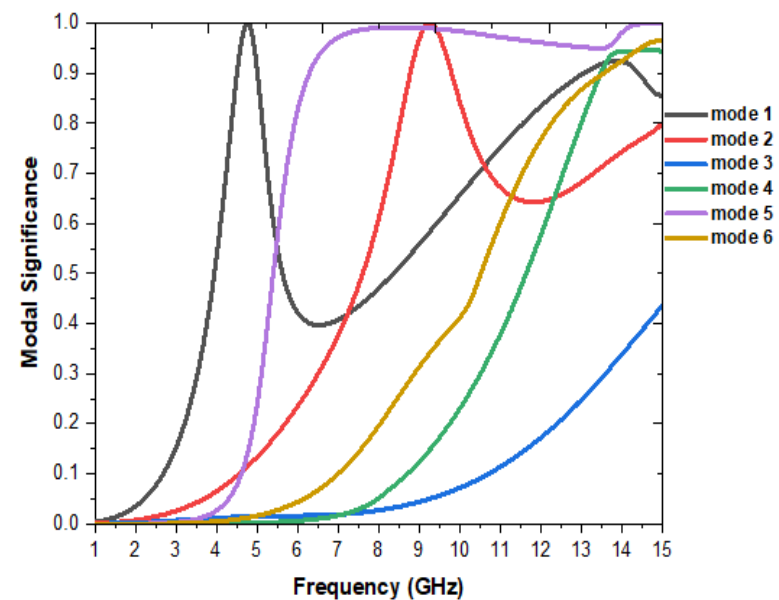

Fig. 5. Modal significance of the first six CMs of antenna-2.
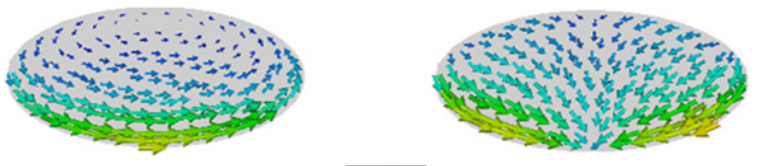

mode 1

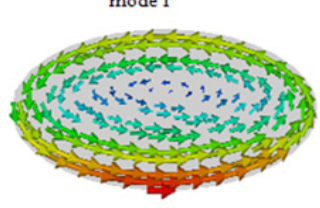

mode 3

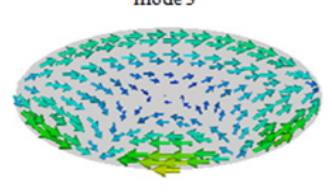

mode 5

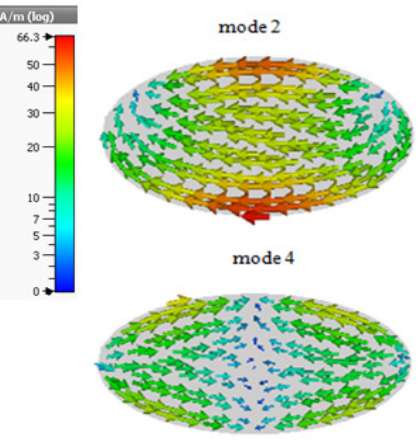

mode 6

Fig. 6. Surface currents of the first six CMs of antenna-2.

- Mode 2 has a current density at the bottom of the ellipse and flows in the opposite direction to the null current.

- Mode 3 forms the current loop and indicates that this mode is not involved in the radiation.

- Mode 4 has a current distribution over the entire area of the ellipse in the horizontal direction. Null currents are present in the upper left and right angles.

- In mode 5 , null is in the middle of the ellipse and medium current is present at the lower center.

- In mode 6, the current flow along the $\mathrm{x}$-axis is split about null in the middle of the $y$-axis in the opposite direction.

Antenna-1 and antenna-2 are not suitable for wideband operation because the modes do not have common surface current areas for excitation. Therefore, to reconfigure the surface currents, the geometry of the new antenna- 3 is formed by subtracting the radiating patch on antenna- 2 from the radiating patch on antenna-1; then the resulting structure is rotated $90^{\circ}$. The proposed elliptical ring antenna is presented in Fig. 7.

The MS of the first six modes of antenna-3 is shown in Fig. 8. Here, only three modes 1,2 , and 4 are significant modes that 


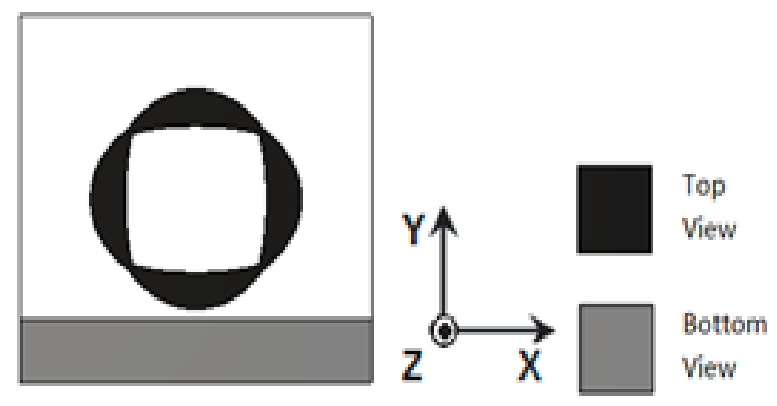

Fig. 7. Geometry of antenna-3.

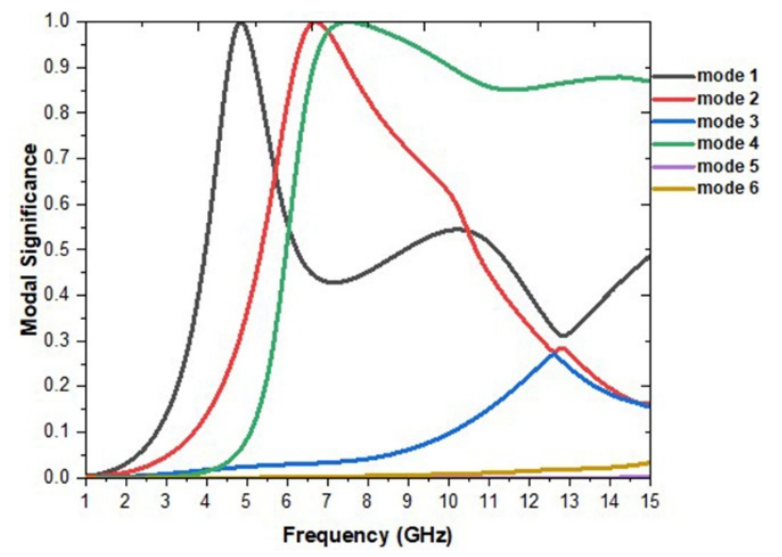

Fig. 8. Modal significance of the first six CMs of antenna-3.

resonate at frequencies of $4.8,6.7$, and $7.54 \mathrm{GHz}$. Other modes are not significant. Unlike the surface current distributions of Figs. 3 and 6, Fig. 9 shows that the current distributions are concentrated in the four corners of the proposed antenna. The currents at four corners are described as follows:

- At the top left corner: the current flow of the three modes 1, 2 , and 4 are in one direction.

- At the top right corner: the current flow of modes 1 and 4 is
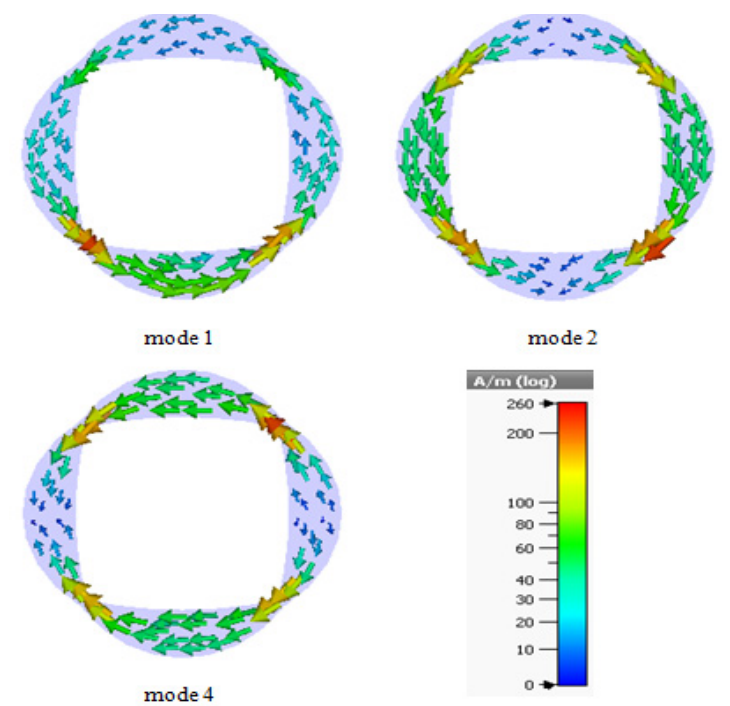

Fig. 9. Surface currents of modes 1, 2, and 4 at frequencies of 4.8 $\mathrm{GHz}, 6.7 \mathrm{GHz}$, and $7.54 \mathrm{GHz}$ of antenna-3. in the same direction, but for mode 2 , it is the opposite.

- At the bottom left corner: the current directions of modes 1 and 2 are the same, but for mode 4, it is the opposite.

- At the bottom right corner: the current directions of modes 2 and 4 are the same, but for mode 1 , it is the opposite.

Through analysis of the surface currents of the CMs of the proposed antenna, four corners were found to be suitable for arranging the feed line in order to excite the desired modes. The bottom right corner of the radiating patch on antenna- 3 was selected for placement of the microstrip feed line. This feed line had a width of $1.7 \mathrm{~mm}$ and length of $6 \mathrm{~mm}$ for wideband characteristics, as shown in Fig. 10. So that wider bandwidth can be achieved [18, 19], exciting multiple modes at their common current maximum with a suitable feeding technique and when they are proximally close enough is desirable.

Adding a microstrip line to antenna- 3 creates a new mode 6 at $10 \mathrm{GHz}$ along with modes 1,2 , and 4 . This mode is called the feed mode. The modal significance of these modes, whose resonance frequencies are close to each other, is presented in Fig. 11. Fig. 12 show the surface current distributions for antenna-3 in the presence of the feed line. Fig. 12 shows that three modes 1,2 , and 6 have a similar current distribution through the feed line at their resonant frequencies of 4.8, 6.7, and $10 \mathrm{GHz}$. Fig. 13

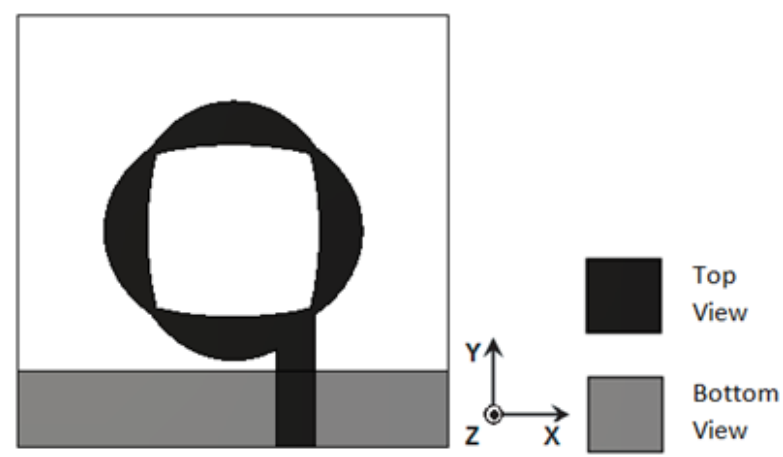

Fig. 10. Geometry of antenna-3 with a microstrip line.

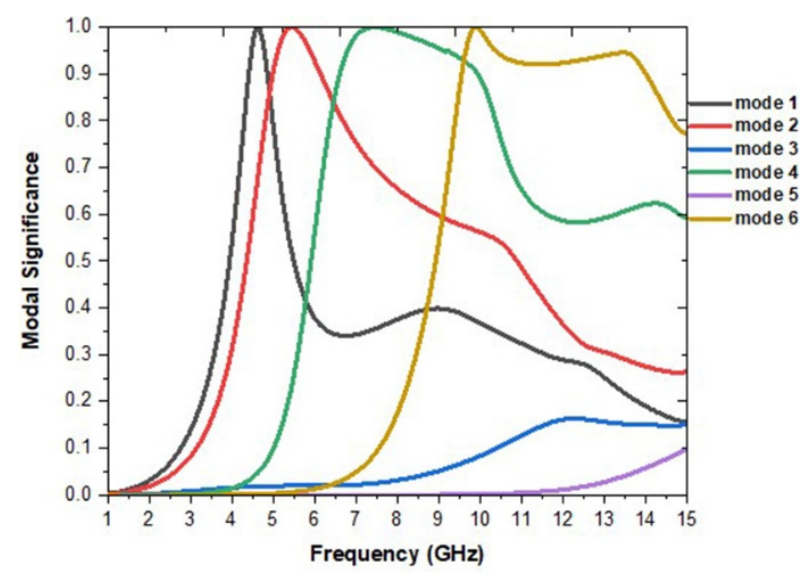

Fig. 11. Modal significance of the first six CMs of antenna-3 with a microstrip feed line. 

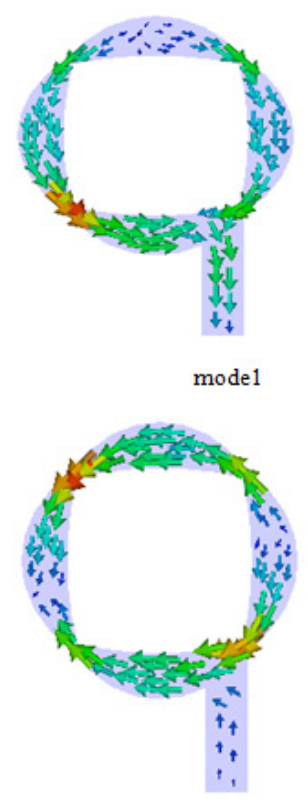

mode 4

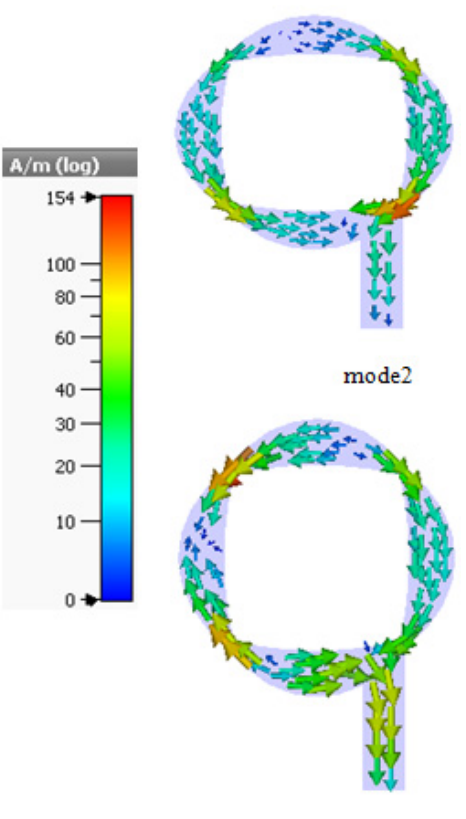

mode 6

Fig. 12. Surface currents of modes $1,2,4$, and 6 at frequencies of $4.6,5.4,7.4$, and $10 \mathrm{GHz}$ of antenna- 3 with a microstrip line.

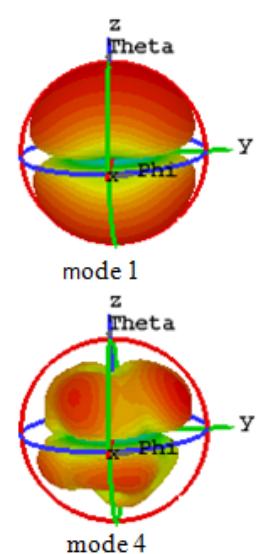

mode 4

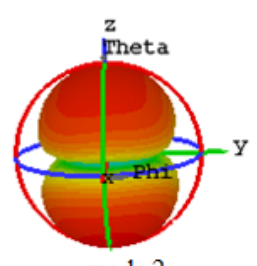

mode 2

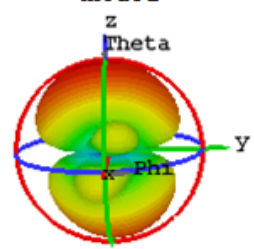

mode 5

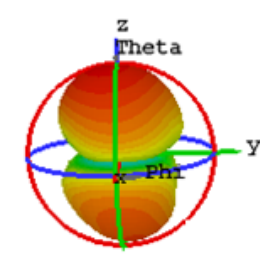

mode 3

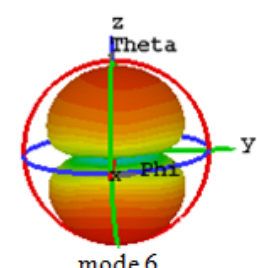

mode 6

Fig. 13. The 3D patterns of first six CMs of antenna-3.

shows that all modes have the maximum radiation in the $\mathrm{z}^{-}$ direction, with the exception of mode 4 , (the null current in the z-direction). As shown in Figs. 11-13, modes 1, 2, and 6 are significant modes and are likely to be excited due to their large MS values, similar current distribution on the feed line, and maximum radiation in the $\mathrm{z}$-direction. Therefore, these three modes contribute more radiation in wideband operation. However, mode 4 is significant due to its large MS value, contributing less radiation to the total radiation of the antenna.

\section{WidEBAND ANTENNA DESIGN}

Characteristic mode analysis (CMA) can also provide insight into the physical aspects of the radiating patch. CMA also allows for change in the size and shape of the patch, offering use-

ful information regarding the optimization of the feed position of the basic antennas. By analyzing the modal significance of $\mathrm{CMs}$ and their surface currents, the four corners of the proposed structure are found to be well suited for the excitation of the desired modes. The same return loss $S_{11}$ is obtained by placing the microstrip feed line at the four corners with the same dimensions of the substrate, feed line, and ground plane. Therefore, the microstrip line is considered properly selected at the bottom left corner of the proposed patch.

The geometry of the proposed antenna consists of an elliptical patch ring, microstrip feed line $\left(F_{W} \times F_{L}\right)$, and the ground plane on the top and bottom of the substrate $\left(W_{S} \times L_{S}\right)$ as shown in Fig. 14. The proposed antenna is printed on an FR-4 substrate of $18 \mathrm{~mm} \times 20 \mathrm{~mm} \times 1.6 \mathrm{~mm}, \varepsilon_{r}=4.3$ and $\tan \delta=$ 0.025 as shown in Fig. 15. The dimensions of the wideband monopole antenna are presented in Table 1.

\section{Parametric Study}

A parametric study was conducted to understand the performance of some parameters of the proposed design. The effect of

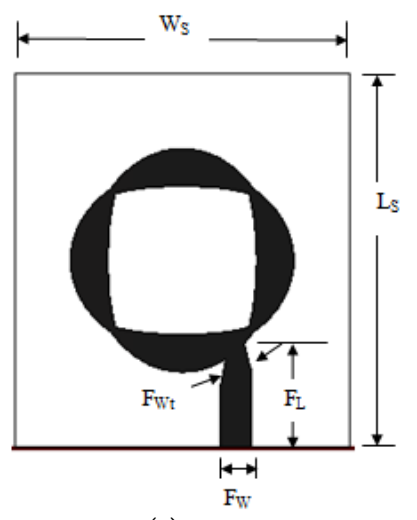

(a)

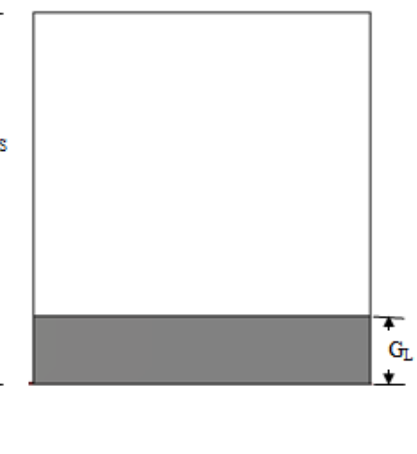

(b)
Fig. 14. Geometry of the proposed wideband monopole antenna: (a) top view and (b) bottom view.

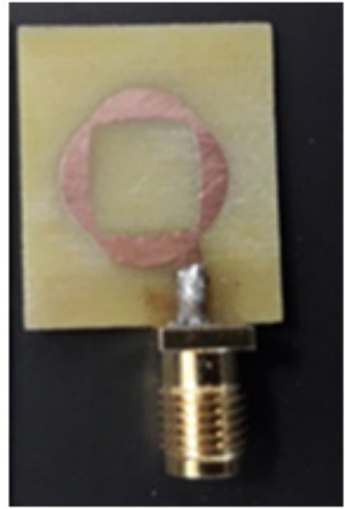

(a)

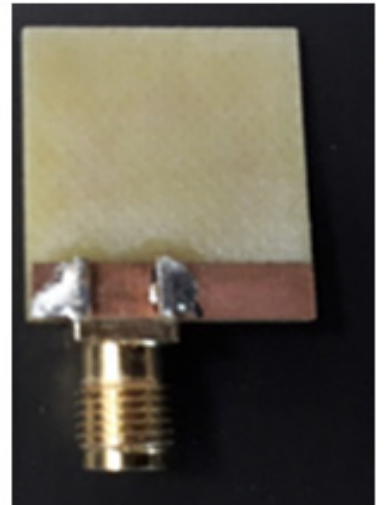

(b)
Fig. 15. Photograph of the fabricated elliptical ring-shaped monopole wideband antenna: (a) top view and (b) bottom view. 
Table 1. Dimensions of the proposed antenna

\begin{tabular}{cc}
\hline Parameter & Value $(\mathrm{mm})$ \\
\hline$W_{s}$ & 18 \\
$L_{s}$ & 20 \\
$G_{L}$ & 6.3 \\
$F_{W}$ & 1.7 \\
$F_{L}$ & 0.6 \\
$F_{W t}$ & 0.6 \\
\hline
\end{tabular}

the tapered microstrip feed line and the length of the ground plane, $G_{L}$, on the return loss parameter, $S_{11}$ were considered as discussed below.

Fig. 16 shows the variation in the width of the tapered microstrip feed line, $F_{W t}$, from 0.2 to $1.0 \mathrm{~mm}$ and its effect on the return loss, $S_{11}$. The $F_{W t}$ value increases, the second resonant frequency shifts towards a higher frequency, while it decreases, the required bandwidth is smaller and the return loss does not fall below $-10 \mathrm{~dB}$. In both cases, the first resonant frequency does not significantly change. When $F_{W t}=0.6 \mathrm{~mm}$, the antenna has good return losses with a wide bandwidth. Therefore, $F_{W t}=0.6$ $\mathrm{mm}$ is chosen as the finest value of ground length over the operating frequency.

Fig. 17 shows the effect of a tapered microstrip line on the reflection coefficient. The proposed antenna is effectively excited by tapering the microstrip line (compared with no tapering) to achieve good return loss characteristics that provide a wide impedance bandwidth.

The effect of changing the ground length $\left(G_{L}\right)$ on the return loss is shown in Fig. 18. With $G_{L}=6.3 \mathrm{~mm}$, the antenna exhibits good return losses with a wide bandwidth. To decrease the value of $G_{L}$, the higher band moves toward a lower band of frequency and return loss does not fall below $-10 \mathrm{~dB}$. While in-

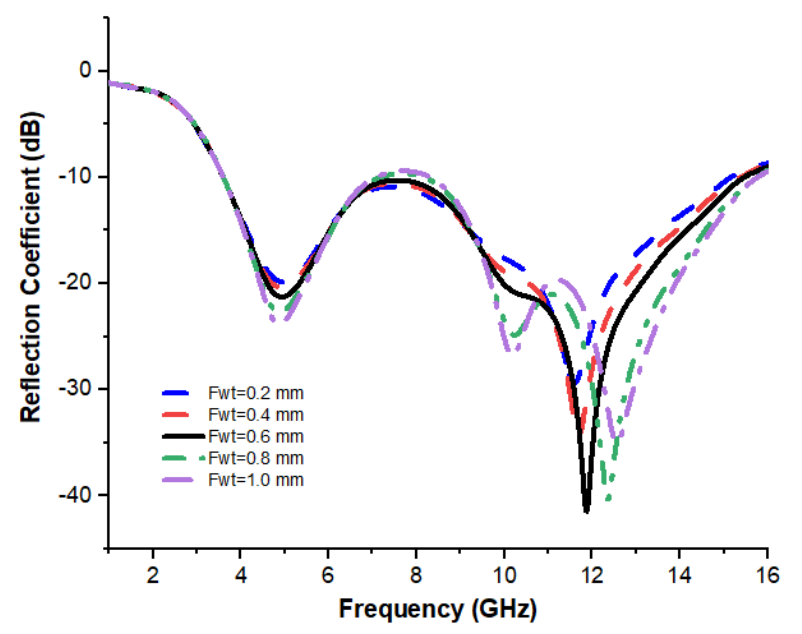

Fig. 16. Effect of the tapered microstrip line width $F_{W t}$ on the reflection coefficient.

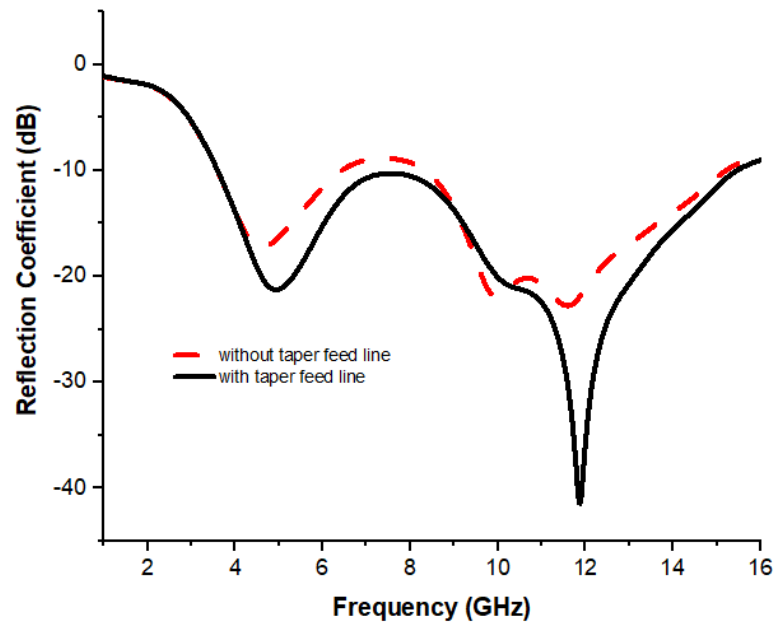

Fig. 17. Reflection coefficient of the proposed antenna with and without tapered microstrip feed line.

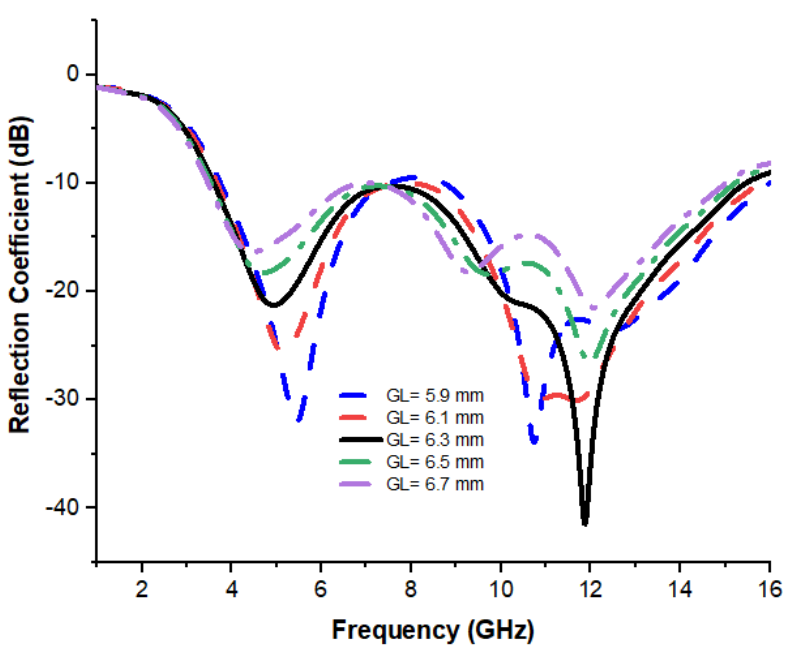

Fig. 18. Effect of the ground length $G_{L}$ on the reflection coefficient.

creasing, the return loss is smaller and the required bandwidth decreases. Therefore, ground length $G_{L}=6.3 \mathrm{~mm}$ is chosen as the finest value to achieve wide bandwidth.

\section{RESULTS AND DISCUSSION}

The network analyzer (Anritsu MS2037C/2) provided the measurement of electrical parameters of the proposed antenna. The wideband characteristics of the monopole antenna are discussed here.

The simulated and measured results of the return loss and gain of the elliptical ring wideband monopole antenna with good agreement are shown in Figs. 19 and 20. Fig. 19 describes the way that the lower resonant frequency of $5 \mathrm{GHz}$ is almost the same in both cases, but the higher resonant frequency of 12 $\mathrm{GHz}$ shifts to a higher frequency compared to the simulation. The gain of the proposed model is maintained constant over the operating frequency range. 


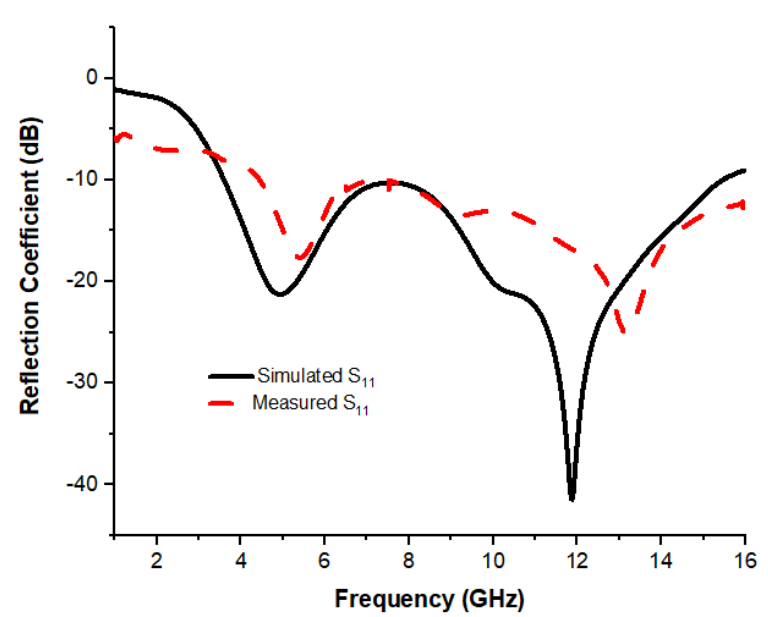

Fig. 19. Simulated and measured results of reflection coefficient.

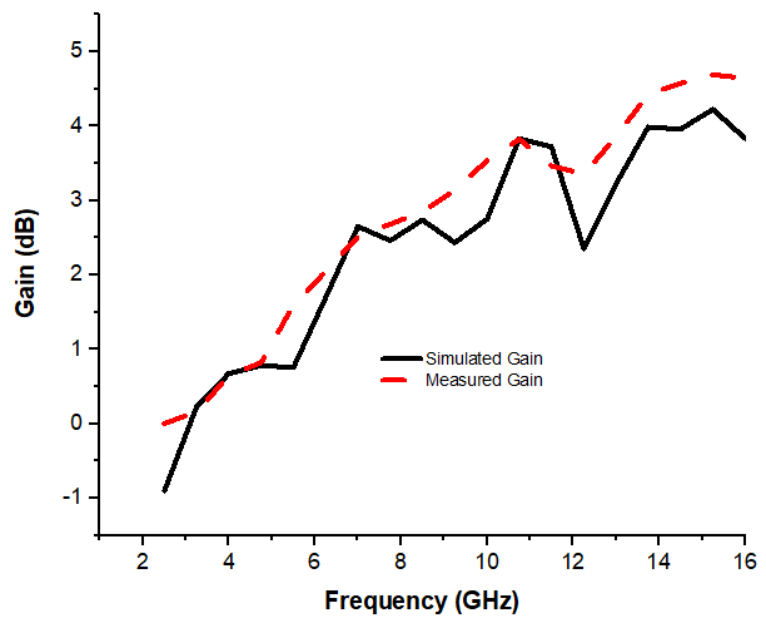

Fig. 20. Simulated and measured results of gain.

The radiation patterns of $\mathrm{H}$-plan and $\mathrm{E}$-plane are presented at $5 \mathrm{GHz}$ and $12 \mathrm{GHz}$ resonant frequencies, respectively, as shown in Fig. 21. At higher frequencies, the radiation patterns are distorted due to higher order modes.

As shown in Table 2, the antenna size, number of modes excited, bandwidth, and gain of the proposed work are compared with the performance of existing antennas provided in existing literature [14-16].

\section{CONCLUSION}

A new elliptical ring-shaped monopole wideband antenna was designed using CMA. The modal significance, surface currents, and modal patterns of first six CMs of the antenna structure were analyzed. The optimal feed position for driving multiple modes such as lower and higher order modes has been identified; a microstrip feed line was placed at respective current maximum to excite for wide bandwidth. The proposed antenna was shown to have a large impedance bandwidth of $124.4 \%$ in the range from 3.6 to $15.46 \mathrm{GHz}$, covering various wireless applications such as $\mathrm{Wi}-\mathrm{Fi}$ and satellite communication.

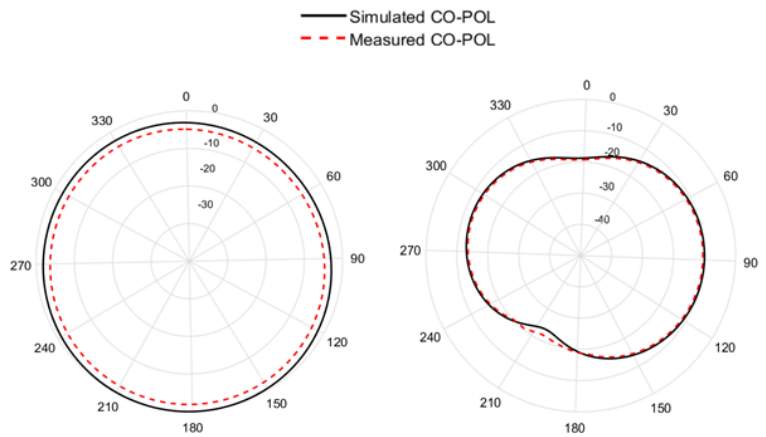

(a)

(b)

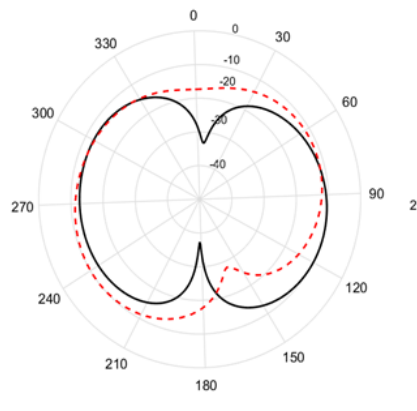

(c)

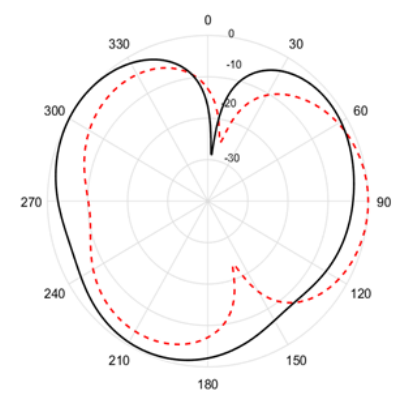

(d)
Fig. 21. Radiation patterns: (a) $5 \mathrm{GHz}$ for E-plane, (b) $5 \mathrm{GHz}$ for $\mathrm{H}$-plane, (c) $12 \mathrm{GHz}$ for E-plane, and (d) $12 \mathrm{GHz}$ for $\mathrm{H}-$ plane.

Table 2. Performance comparison between proposed work and existing antenna designs

\begin{tabular}{lccccc}
\hline Study & $\begin{array}{c}\text { Size } \\
\left(\mathrm{mm}^{2}\right)\end{array}$ & $\begin{array}{c}\text { No. of } \\
\text { modes } \\
\text { excited }\end{array}$ & $\begin{array}{c}\text { Bandwidth } \\
\left(S_{11}<-10 \mathrm{~dB}\right) \\
(\mathrm{GHz})\end{array}$ & $\begin{array}{c}\% \\
\text { BW }\end{array}$ & $\begin{array}{c}\text { Gain } \\
(\mathrm{dB})\end{array}$ \\
\hline$[14]$ & $36 \times 35$ & 2 & $2.3-3.9$ & 51.6 & 4 \\
{$[15]$} & $80 \times 35$ & 2 & $1.85-2.9$ & 44.2 & 4 \\
{$[16]$} & $140.17 \times 134.54$ & 3 & $2.86-8.16$ & 96 & - \\
Proposed & $20 \times 18$ & 3 & $3.6-15.46$ & 124.4 & 4 \\
\hline
\end{tabular}

\section{REFERENCES}

[1] K. L. Wong, Compact and Broadband Microstrip Antennas. New York, NY: John Wiley \& Sons, 2002.

[2] F. Yang, X. X. Zhang, X. Ye, and Y. Rahmat-Samii, "Wideband E-shaped patch antennas for wireless communications," IEEE Transactions on Antennas and Propagation, vol. 49, no. 7, pp. 1094-1100, 2001.

[3] M. A. Matin, B. S. Sharif, and C. C. Tsimenidis, "Probe fed stacked patch antenna for wideband applications," IEEE Transactions on Antennas and Propagation, vol. 55, no. 8, pp. 2385-2388, 2007.

[4] P. Khanna, A. Sharma, A. K. Singh, and A. Kumar, "A 
CPW-fed octagonal ring shaped wide band antenna for wireless applications," Advanced Electromagnetics, vol. 7, no. 3, pp. 87-92, 2018.

[5] R. J. Garbacz, "Modal expansions for resonance scattering phenomena," Proceedings of the IEEE, vol. 53, no. 8, pp. 856-864, 1965.

[6] R. Harrington and J. Mautz, "Theory of characteristic modes for conducting bodies," IEEE Transactions on Antennas and Propagation, vol. 19, no. 5, pp. 622-628, 1971.

[7] R. Harrington and J. Mautz, "Computation of characteristic modes for conducting bodies," IEEE Transactions on Antennas and Propagation, vol. 19, no. 5, pp. 629-639, 1971.

[8] M. Cabedo-Fabres, E. Antonino-Daviu, A. Valero-Nogueira, and M. F. Bataller, "The theory of characteristic modes revisited: a contribution to the design of antennas for modern applications," IEEE Antennas and Propagation Magazine, vol. 49, no. 5, pp. 52-68, 2007.

[9] J. B. Kamili and A. Bhattacharya, "Design of a novel compact bowtie antenna and analysis using characteristic modes," in Proceedings of 2019 IEEE Region 10 Conference (TENCON), Kochi, India, 2019, pp. 1903-1907.

[10] E. Antonino-Daviu, M. Cabedo-Fabres, M. Sonkki, N. M. Mohamed-Hicho, and M. Ferrando-Bataller, "Design guidelines for the excitation of characteristic modes in slotted planar structures," IEEE Transactions on Antennas and Propagation, vol. 64, no. 12, pp. 5020-5029, 2016.

[11] B. Ganji and R. H. Shaik, "Characteristic mode analysis based X-band defected dodecagonal patch antenna," International Journal of Engineering and Advanced Technology (IJEAT), vol. 9, no.1, pp. 3216-3220, 2019.

[12] P. Sumithra and D. Kannadassan, "Characteristic mode analysis of concentric circular ring antenna," in Proceedings of 2018 3rd International Conference on Communication

\section{Bhaskara Rao Perli}

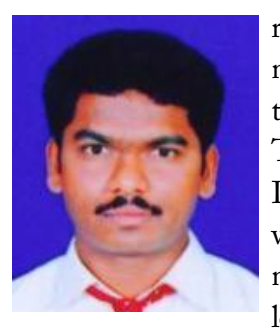

received his B.Tech. degree in electronics and communication engineering and M.Tech. degree in systems and signal processing from Jawaharlal Nehru Technological University, Hyderabad, Telangana, India, in 2005 and 2011, respectively. He is currently working toward his $\mathrm{Ph} . \mathrm{D}$. in electronics and communication engineering at the Jawaharlal Nehru Technological University, Anantapur, Ananthapuramu, Andhra Pradesh, India. His research interests include microstrip antennas, microwave antennas, and wireless communications. and Electronics Systems (ICCES), Coimbatore, India, 2018, pp. 1037-1040.

[13] C. Wang, Y. Chen, and S. Yang, "Bandwidth enhancement of a dual-polarized slot antenna using characteristic modes," IEEE Antennas and Wireless Propagation Letters, vol. 17, no. 6, pp. 988-992, 2018.

[14] D. Wen, Y. Hao, H. Wang, and H. Zhou, "Design of a wideband antenna by manipulating characteristic modes of a metallic loop," Microwave and Optical Technology Letters, vol. 61, no. 2, pp. 513-518, 2019.

[15] D. Wen, Y. Hao, H. Wang, and H. Zhou, "Design of a wideband antenna with stable omnidirectional radiation pattern using the theory of characteristic modes," IEEE Transactions on Antennas and Propagation, vol. 65, no. 5, pp. 2671-2676, 2017.

[16] M. M. Elsewe and D. Chatterjee, "Ultra-wide bandwidth enhancement of single-layer single-feed patch antenna using the theory of characteristic modes," Applied Computational Electromagnetics Society Journal, vol. 33, no. 3, pp. 363-366, 2018.

[17] Y. Chen and C. F. Wang, Characteristic Modes: Theory and Applications in Antenna Engineering. Hoboken, NJ: John Wiley \& Sons, 2015.

[18] Y. Chen and C. F. Wang, "From antenna design to feeding design: a review of characteristic modes for radiation problems," in Proceedings of the 2013 International Symposium on Antennas \& Propagation, Nanjing, China, 2013, pp. 68-70.

[19] J. Rahola, D. Ludick, and P. Futter, "Characteristic modes and antenna bandwidth," in Proceedings of 2014 IEEE Antennas and Propagation Society International Symposium (APSURSI), Memphis, TN, 2014, pp. 1415-1416.

Maheswara Rao Avula

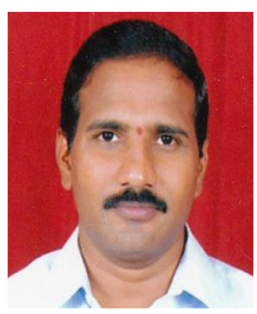

received his B.E. degree in electronics and communication engineering from Andhra University, Visakhapatnam, India, in 1995, his M.E. degree in applied electronics from Madras University, Chennai, India, in 2000, and his Ph.D. in wireless communications from Jawaharlal Nehru Technological University, Anantapur, Andhra Pradesh, India. He is currently an associate professor in the Department of

Electronics and Communication Engineering, PBR Visvodaya Institute of Technology \& Science, Kavali, Andhra Pradesh, India. His current research interests include wireless data networks, microwave antennas, microstrip antennas, and wireless communications. 